\title{
Modeling Topic and Role Information in Meetings Using the Hierarchical Dirichlet Process
}

\author{
Songfang Huang and Steve Renals \\ The Centre for Speech Technology Research \\ University of Edinburgh, Edinburgh, EH8 9LW, UK \\ $\{$ s.f.huang, s.renals\}@ed.ac.uk
}

\begin{abstract}
In this paper, we address the modeling of topic and role information in multiparty meetings, via a nonparametric Bayesian model called the hierarchical Dirichlet process. This model provides a powerful solution to topic modeling and a flexible framework for the incorporation of other cues such as speaker role information. We present our modeling framework for topic and role on the AMI Meeting Corpus, and illustrate the effectiveness of the approach in the context of adapting a baseline language model in a large-vocabulary automatic speech recognition system for multiparty meetings. The adapted LM produces significant improvements in terms of both perplexity and word error rate.
\end{abstract}

\section{Introduction}

A language model (LM) aims to provide a predictive probability distribution for the next word based on a history of previously observed words. The $n$-gram model, which forms the conventional approach to language modeling in stateof-the-art automatic speech recognition (ASR) systems, simply approximates the history as the immediately preceding $n-1$ words. Although this has been demonstrated to be a simple but effective model, the struggle to improve over it continues. Broadly speaking, such attempts focus on the improved modeling of word sequences, or on the incorporation of richer knowledge. Approaches which aim to improve on maximum likelihood $n$-gram models of word sequences include neural network-based models [1, latent variable models [2], and a Bayesian framework 34]. The exploitation of richer knowledge has included the use of morphological information in factored LMs [5], syntactic knowledge using structured LMs [6], and semantic knowledge such as topic information using Bayesian models [7].

In this paper, we investigate language modeling for ASR in multiparty meetings through the inclusion of richer knowledge in a conventional $n$-gram language model. We have used the AMI Meeting Corpus [1], which consists of 100 hours of multimodal meeting recordings with comprehensive annotations at a number

1 http://corpus.amiproject.org

A. Popescu-Belis and R. Stiefelhagen (Eds.): MLMI 2008, LNCS 5237, pp. 214225 2008.

(C) Springer-Verlag Berlin Heidelberg 2008 
of different levels. About $70 \%$ of the corpus was elicited using a design scenario, in which the four participants play the roles of project manager (PM), marketing expert (ME), user interface designer (UI), and industrial designer (ID), in an electronics company that decides to develop a new type of television remote control. Our work in this paper is motivated by the fact that the AMI Meeting Corpus has a wealth of multimodal information such as audio, video, lexical, and other high-level knowledge. From the viewpoint of language modeling, the question for us is whether there are cues beyond lexical information which can help to improve an $n$-gram LM. If so, then what are those cues, and how can we incorporate them into an $n$-gram LM? To address this question, we here focus on the modeling of topic and role information using a hierarchical Dirichlet process 9 .

Consider an augmented $n$-gram model for ASR, with its context enriched by the inclusion of two cues from meetings: the topic and the speaker role. Unlike role, which could be seen as deterministic information available in the corpus, topic refers to the semantic context, which is typically extracted by an unsupervised approach. One popular topic model is latent Dirichlet allocation (LDA) 10, which can successfully find latent topics based on the co-occurrences of words in a 'document'. However, there are two difficulties arising from the application of LDA to language modeling of multiparty conversational speech. First, it is important to define the notion of document to which the LDA model can be applied: conversational speech consists of sequences of utterances, which do not comprise well-defined documents. Second, it is not easy to decide the number of topics in advance, a requirement for LDA.

The hierarchical Dirichlet process (HDP) 9 is a nonparametric generalization of LDA which extends the standard LDA model in two ways. First, the HDP uses a Dirichlet process prior for the topic distribution, rather than the Dirichlet distribution used in LDA. This enables the HDP to determine the number of topics required. Second, the hierarchical tree structure enables the HDP to share mixture components (topics) between groups of data. In this paper we exploit the HDP as our modeling approach for automatic topic learning. Moreover, we also find it easier to incorporate roles together with topics by expressing them as an additional level of variables into the HDP hierarchy.

Some previous work has been done in the area of combining $n$-gram models and topic models such as LDA and probabilistic latent semantic analysis (pLSA) for ASR on different data, for example, broadcast news [11]12, lecture recordings [13, and Japanese meetings [14. The new ideas we exploit in this work cover the following aspects. First, we use the nonparametric HDP for topic modeling to adapt $n$-gram LMs. Second, we consider sequential topic modeling, and define documents for the HDP by placing a moving window over the sequences of short sentences. Third, we incorporate the role information with topic models in a hierarchical Bayesian framework. In the rest of this paper, we will review topic models, and introduce our framework for modeling topic and role information using the HDP, followed by a set of perplexity and word error rate (WER) experiments. 


\section{Probabilistic Topic Model}

Topic models, which have received a growing interest in the machine learning community, are used in document modeling to find a latent representation connecting documents and words - the topic. In a topic model, words in a document exchangeably co-occur with each other according to their semantics, following the "bag-of-words" assumption.

Suppose there are $D$ documents in the corpus, and $W$ words in the vocabulary. Each document $d=1, \ldots, D$ in the corpus is represented as a mixture of latent topics, with the mixing proportions over topics denoted by $\boldsymbol{\theta}_{d}$. Each topic $k=$ $1, \ldots, K$ in turn is a multinomial distribution over words in the vocabulary, with the vector of probabilities for words in topic $k$ denoted by $\phi_{k}$.

In this section, we review two "bag-of-word" models, LDA and the HDP, following Teh et al. 91516.

\subsection{Latent Dirichlet Allocation}

Latent Dirichlet allocation [10] is a three-level hierarchical Bayesian model, which pioneered the use of the Dirichlet distribution for latent topics. That is, the

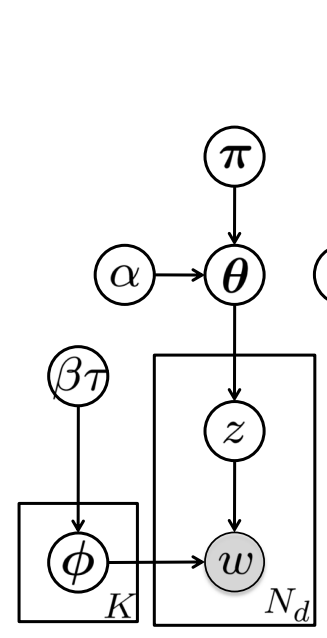

(A)

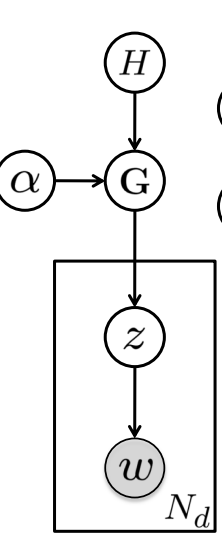

(B)

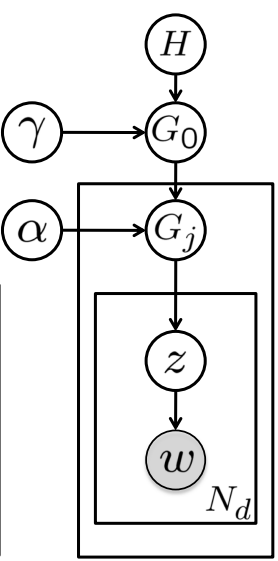

(C)

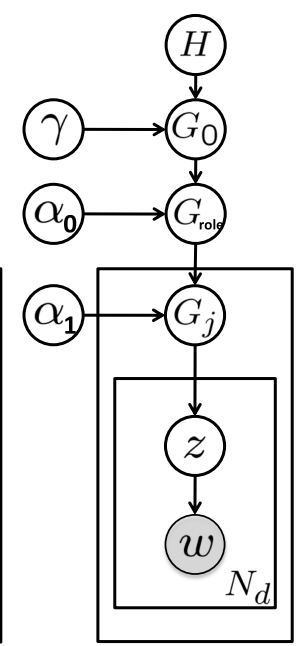

(D)

Fig. 1. Graphical model depictions for (A) latent Dirichlet allocation (finite mixture model), (B) Dirichlet process mixture model (infinite mixture model), (C) 2-level hierarchical Dirichlet process model, and (D) the role-HDP where $G_{\text {role }}$ denotes the DP for one of the four roles (PM, ME, UI, and ID) in the AMI Meeting Corpus. Each node in the graph represents a random variable, where shading denotes an observed variable. Arrows denote dependencies among variables. Rectangles represent plates, or repeated sub-structures in the model. 
topic mixture weights $\boldsymbol{\theta}_{d}$ for the $d$ th document are drawn from a prior Dirichlet distribution with parameters $\alpha, \boldsymbol{\pi}$ :

$$
P\left(\boldsymbol{\theta}_{d} \mid \alpha \boldsymbol{\pi}\right)=\frac{\Gamma\left(\sum_{i=1}^{K} \alpha \pi_{i}\right)}{\prod_{i=1}^{K} \Gamma\left(\alpha \pi_{i}\right)} \theta_{1}^{\alpha \pi_{1}-1} \ldots \theta_{K}^{\alpha \pi_{K}-1}
$$

where $K$ is the predefined number of topics in LDA, $\Gamma$ is the Gamma function, $\alpha \boldsymbol{\pi}=\left\{\alpha \pi_{1}, \ldots, \alpha \pi_{K}\right\}$ represents the prior observation counts of the $K$ latent topics with $\alpha \pi_{i}>0: \pi$ is the corpus-wide distribution over topics, and $\alpha$ is called the concentration parameter which controls the amount of variability from $\boldsymbol{\theta}_{d}$ to their prior mean $\pi$.

Similarly, Dirichlet priors are placed over the parameters $\phi_{k}$ with the parameters $\beta \tau$. We write:

$$
\boldsymbol{\theta}_{d}\left|\boldsymbol{\pi} \sim \operatorname{Dir}(\alpha \boldsymbol{\pi}) \quad \boldsymbol{\phi}_{k}\right| \boldsymbol{\tau} \sim \operatorname{Dir}(\beta \boldsymbol{\tau})
$$

Fig. 1.(A) depicts the graphical model representation for LDA. The generative process for words in each document is as follows: first draw a topic $k$ with probability $\theta_{d k}$, then draw a word $w$ with probability $\phi_{k w}$. Let $w_{i d}$ be the $i$ th word token in document $d$, and $z_{i d}$ the corresponding drawn topic, then we have the following multinomial distributions:

$$
z_{i d}\left|\boldsymbol{\theta}_{d} \sim \operatorname{Mult}\left(\boldsymbol{\theta}_{d}\right) \quad w_{i d}\right| z_{i d}, \phi_{z_{i d}} \sim \operatorname{Mult}\left(\phi_{z_{i d}}\right)
$$

\subsection{Hierarchical Dirichlet Process}

LDA uses Dirichlet distributed latent variables to represent shades of memberships to different cluster or topics. In the HDP nonparametric models are used to avoid the need for model selection [16. Two extensions are made in the HDP: first the Dirichlet distributions in LDA are replaced by Dirichlet processes in the HDP as priors for topic proportions; second, the priors are arranged in a tree structure.

Dirichlet Process. The Dirichlet process (DP) is a stochastic process, first formalised in [17] for general Bayesian modeling, which has become an important prior for nonparametric models. Nonparametric models are characterised by allowing the number of model parameters to grow with the amount of training data. This helps to alleviate over- or under-fitting problems, and provides an alternative approach to parametric model selection or averaging.

A random distribution $G$ over a space $\Theta$ is called a Dirichlet process distributed with base distribution $H$ and concentration parameter $\alpha$, if

$$
\left(G\left(A_{1}\right), \ldots, G\left(A_{r}\right)\right) \sim \operatorname{Dir}\left(\alpha H\left(A_{1}\right), \ldots, \alpha H\left(A_{r}\right)\right)
$$

for every finite measurable partition $A_{1}, \ldots, A_{r}$ of $\Theta$. We write this as $G \sim$ $\operatorname{DP}(\alpha, H)$. The parameter $H$, a measure over $\Theta$, is intuitively the mean of the DP. The parameter $\alpha$, on the other hand, can be regarded as an inverse variance 
of its mass around the mean $H$, with larger values of $\alpha$ for smaller variances. More importantly in infinite mixture models, $\alpha$ controls the expected number of mixture components in a direct manner, with larger $\alpha$ implying a larger number of mixture components a priori.

Draws from a DP are composed as a weighted sum of point masses located at the previous draws $\theta_{1}, \ldots, \theta_{n}$. This leads to a constructive definition of the DP called the stick-breaking construction [18]:

$$
\beta_{k} \sim \operatorname{Beta}(1, \alpha) \quad \pi_{k}=\beta_{k} \prod_{l=1}^{k-1}\left(1-\beta_{k}\right) \quad \theta_{k}^{*} \sim H \quad G=\sum_{k=1}^{\infty} \pi_{k} \delta_{\theta_{k}^{*}}
$$

Then $G \sim \operatorname{DP}(\alpha, H)$. $\theta_{k}^{*}$ is a unique value among $\theta_{1}, \ldots, \theta_{n}$, and $\delta_{\theta_{k}^{*}}$ denotes a point mass at $\theta_{k}^{*}$. The construction of $\pi$ can be understood as follows [15. Starting with a stick of length 1 , first break it at $\beta_{1}$, assign $\pi_{1}$ to be the length of stick just broken off. Then recursively break the other portion to obtain $\pi_{2}, \pi_{3}$ and so forth. The stick-breaking distribution over $\boldsymbol{\pi}$ is sometimes written as $\pi \sim \operatorname{GEM}(\alpha))^{2}$, and satisfies $\sum_{k=1}^{\infty} \pi_{k}=1$ with probability one. This definition is important for the inference for the DP.

Recall in Equation 2 for LDA, a finite-dimensional Dirichlet distribution (i.e., in which $\pi$ is a $K$-dimensional vector) is used as prior for distribution of topic proportions. LDA, in this sense, is a finite mixture model. If we use a DP instead as prior for mixing topic proportions, that is, $\boldsymbol{\theta}_{d} \sim \operatorname{DP}(\alpha, H)$ where $\phi_{k} \mid H \sim$ $\operatorname{Dir}(\beta \boldsymbol{\tau})$, then the stick-breaking construction for $\pi \sim \operatorname{GEM}(\alpha)$ will produce a countably infinite dimensional vector $\boldsymbol{\pi}$. In this way, the number of topics in this DP-enhanced LDA model is potentially infinite, the number of topics increasing with the available data.

This model, as shown in Fig. 1.(B), is called the Dirichlet process mixture model (also known as an infinite mixture model).

Hierarchical Framework. Besides the nonparametric extension of LDA from Dirichlet distribution to Dirichlet process, Teh et al. 9] further extended the Dirichlet process mixture model from a flat structure to a hierarchical structure, called a hierarchical Dirichlet process mixture model. This extended model uses the hierarchical Dirichlet process as priors. Similar to the DP, the HDP is a prior for nonparametric Bayesian modeling. The difference is that in the HDP, it is assumed that there are groups of data, and that the infinite mixture components are shared among these groups.

Considering a simple 2-level HDP as an example, as shown is Fig. 1. (C), the HDP defines a set of random probability measure $G_{j}$, one for each group of data, and a global random probability measure $G_{0}$. The global measure $G_{0}$ is distributed as a DP with concentration parameter $\gamma$ and base probability measure $H$, and the random measure $G_{j}$, assuming conditionally independent given $G_{0}$, are in turn distributed as a DP with concentration parameter $\alpha$ and base probability measure $G_{0}$ :

$$
G_{0}\left|\gamma, H \sim \operatorname{DP}(\gamma, H) \quad G_{j}\right| \alpha, G_{0} \sim \operatorname{DP}\left(\alpha, G_{0}\right)
$$

${ }^{2}$ GEM stands for Griffiths, Engen, and McCloskey. 
This results in a hierarchy of DPs, in which their dependencies are specified by arranging them in a tree structure. Although this is a 2-level example, the HDP can readily be extended to as many levels as required.

An HDP-enhanced LDA model, therefore, will have a potentially infinite number of topics, and these topics will be shared among groups of data. If an HDP is used as a prior for topic modeling, then the baseline distribution $H$ provides the prior distribution for words in the vocabulary, i.e., $\phi_{k} \mid H \sim \operatorname{Dir}(\beta \boldsymbol{\tau})$. The distribution $G_{0}$ varies around the prior $H$ with the amount of variability controlled by $\gamma$, i.e., $G_{0} \sim \operatorname{DP}(\gamma, \operatorname{Dir}(\beta \boldsymbol{\tau}))$. The actual distribution $G_{d}$ for $d$ th group of data (words in $d$ th document in topic models) deviates from $G_{0}$, with the amount of variability controlled by $\alpha$, i.e., $G_{d} \sim \operatorname{DP}\left(\alpha, G_{0}\right)$. Together with (3), this completes the definition of an HDP-enhanced LDA topic model.

\section{Modeling Topic and Role Using HDP}

In this section we discuss three key questions concerning the modeling of topic and role using the HDP. First, how should a document be defined in a multiparty meeting? Second, how do we introduce role into the HDP framework? Third, how can the local estimates from an HDP be used to adapt a baseline $n$-gram LM for an ASR system?

Document Definition. The target application of the HDP in this paper is the adaptation of LMs for a multiparty conversational ASR system. For each sentence in the testing data, we need to find a corresponding document for the HDP, based on which topics are extracted, and then the LM is dynamically adapted according to the topic information. Documents also include information about speaker role. In the AMI Meeting Corpus, meetings are manually annotated with word transcription (in *.words.xml), with time information being further obtained via forced alignment. Also available in the corpus are the segment annotations (in *.segments.xml). Role information can be easily determined from the annotations in the corpus. We used the following procedure,

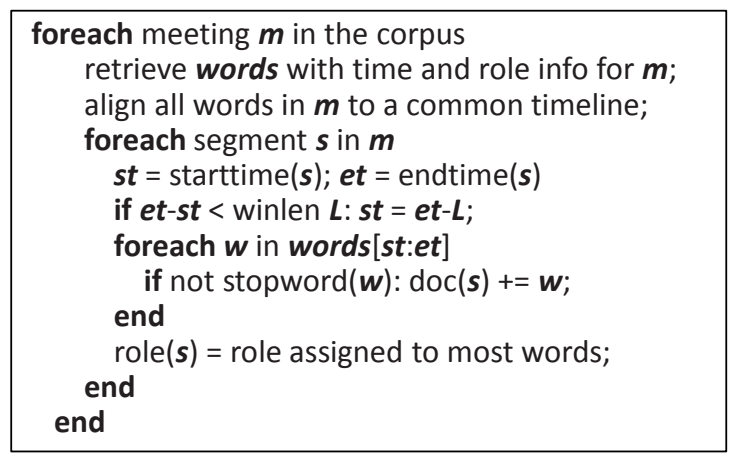

Fig. 2. The procedure used to define documents for the HDP/rHDP 
as shown in Fig. 2, to obtain documents: for each scenario meeting, first align all the words in it along a common timeline; then for each sentence/segment, collect those non-stop words belonging to a window of length $L$, by backtracing from the end time of the sentence/segment, as the document. The role that has been assigned to the most of words in the window is selected as the role for that document.

By collecting all documents for meetings belonging to the training and testing data respectively, we can obtain the training data for HDP model and the testing data for perplexity evaluation. A similar idea applies to finding documents dynamically for ASR experiments. The difference is that we do not have the segment annotations in this case. Instead speech segments, obtained by either automatic or manual approaches, are used as units for finding documents as well as for ASR. In the ASR case we use an online unsupervised method: ASR hypotheses (with errors and time information) from previous segments are used to define documents for HDP inference for the current segment. In both cases above, we simply ignore those segments without corresponding documents.

Incorporation of Role Information. As a preliminary attempt, we consider the problem of introducing role into the HDP hierarchy to enable better topic modeling. In the scenario meetings of the AMI Meeting Corpus, each of the four participants in a meeting series was assigned a different role (PM, ME, UI, or ID). Since different participants have different roles to play, there may be a different topic distribution, and in turn different dominant words, specific to each role. However, we still expect topic models to work as a whole on the corpus rather than having four separate topic models. The HDP is thus an appropriate model, because it has a flexible framework to express DP dependencies using a tree structure.

Documents were defined as described above for those scenario meetings with role information, a one-to-one mapping. We grouped the documents for each of the four roles, and assigned a DP $G_{\text {role }}$ for each role, which then served as the parent DP in the HDP hierarchy (the base probability measure) for all DPs corresponding to documents belonging to that role. To share the topics among four roles, a global DP $G_{0}$ was used as the common base probability measure for the four role DPs $G_{\text {role }}$. See the graphical model shown in Fig. 1. (D) for the HDP hierarchy. Formally speaking, we used a 3-level HDP, referred to as rHDP, to model topic and role information in the AMI Meeting Corpus:

$$
G_{0}\left|\gamma, H \sim \operatorname{DP}(\gamma, H), G_{\text {role }}\right| \alpha_{0}, G_{0} \sim \operatorname{DP}\left(\alpha_{0}, G_{0}\right), G_{j} \mid \alpha_{1}, G_{\text {role }} \sim \operatorname{DP}\left(\alpha_{1}, G_{\text {role }}\right)(7)
$$

Combination with $\boldsymbol{n}$-grams. A topic in an HDP is a multinomial distribution over words in the vocabulary (denoted as $\phi_{k}$ ), which can be considered as a unigram model. To be precise, we use $P_{\text {hdp }}(w \mid d)$ to denote the unigram probabilities obtained by the HDP based on the $j$ th document $d$. The HDP probability $P_{\text {hdp }}(w \mid d)$ is approximated as a sum over all the latent topics $\phi_{k}$ for that document, supposing there are $K$ topics in total in the HDP at the current time: 


$$
P_{\mathrm{hdp}}(w \mid d) \approx \sum_{k=1}^{K} \phi_{k w} \cdot \theta_{d k}
$$

where the probability vector $\phi_{k}$ is estimated during training and remains fixed in testing, while the topic weights $\boldsymbol{\theta}_{d} \mid G_{0} \sim \mathrm{DP}\left(\alpha_{0}, G_{0}\right)$ are document-dependent and thus are calculated dynamically for each document. For rHDP, the difference is that the topic weights are derived from role DPs, i.e., $\boldsymbol{\theta}_{d} \mid G_{\text {role }} \sim \operatorname{DP}\left(\alpha_{1}, G_{\text {role }}\right)$.

As in [19, we treat $P_{\text {hdp }}(w \mid d)$ as a dynamic marginal and use the following equation to adapt the baseline $n$-gram model $P_{\text {back }}(w \mid h)$ to get an adapted $n$ gram $P_{\text {adapt }}(w \mid h)$, where $z(h)$ is a normalisation factor:

$$
P_{\text {adapt }}(w \mid h)=\frac{\alpha(w)}{z(h)} \cdot P_{\text {back }}(w \mid h) \quad \alpha(w) \approx\left(\frac{P_{\text {hdp }}(w \mid d)}{P_{\text {back }}(w)}\right)^{\mu}
$$

\section{Experiment and Result}

We report some experimental results in this section. The HDP was implemented as an extension to the SRILM toolkit 3 . All baseline LMs used here were trained using SRILM, and the N-best generation and rescoring were based on a modified tool from SRILM.

Since we considered the role information, which is only available in scenario AMI meetings, we used part of the AMI Meeting Corpus for our experiments. There are 138 scenario meetings in total, of which 118 were used for training and the other 20 for testing (about 11 hours). We used the algorithm introduced in Section 3 to extract the corresponding document for each utterance. The average number of words in the resulting documents for window lengths of 10 and 20 seconds was 10 and 14 respectively. Data for $n$-gram LMs were obtained as usual for training and testing.

We initialized both HDP and rHDP models with 50 topics, and $\beta=0.5$ for Equation 2. HDP/rHDP models were trained on documents of 10 seconds window length from the scenario AMI meetings with a fixed size vocabulary of 7,910 words, using a Markov Chain Monte Carlo (MCMC) sampling method. The concentration parameters were sampled using the auxiliary variable sample scheme in 9]. We used 3,000 iterations to 'burn-in' the HDP/rHDP models.

\subsection{Perplexity Experiment for LMs}

In order to see the effect of the adapted LMs on perplexity, we trained three baseline LMs: the first one used the AMI $n$-gram training data, the second used the Fisher conversational telephone speech data (fisher-03-p1+p2), and the third used the Hub-4 broadcast news data (hub4-Im96). A fourth LM was trained using all three datasets. All the four LMs were trained with standard parameters using SRILM: trigrams, cut-off value of 2 for trigram counts, modified KneserNey smoothing, interpolated model. A common vocabulary with 56,168 words

\footnotetext{
3 http://www.speech.sri.com/projects/srilm
} 
Table 1. The perplexity results of HDP/rHDP-adapted LMs

\begin{tabular}{|c||c|c|c|}
\hline LMs & Baseline & HDP-adapted & rHDP-adapted \\
\hline \hline AMI & 107.1 & 100.7 & 100.7 \\
Fisher & 228.3 & 176.5 & 176.4 \\
Hub-4 & 316.4 & 248.9 & 248.8 \\
AMI+Fisher+Hub-4 & 172.9 & 144.1 & 143.9 \\
\hline
\end{tabular}

was used for the four LMs, which has 568 out-of-vocabulary (OOV) words for the AMI test data.

The trained HDP and rHDP models were used to adapt the above four baseline $n$-gram models respectively, using Equation 9 with $\mu=0.5$. Different vocabularies were used by the HDP/rHDP models compared with the baseline $n$-gram models. Only those words occurring in both the HDP/rHDP vocabulary and the $n$-gram vocabulary were scaled using Equation 9 . Table 4.1 shows the perplexity results for the adapted $n$-gram models. We can see both HDP- and rHDP-adapted LMs produced significant reduction in perplexity, however there was no significant difference between using the HDP or rHDP as the dynamic marginal in the adaptation.

\subsection{ASR Experiment}

Finally, we investigated the effectiveness of the adapted LMs based on topic and role information from meetings on a practical large vocabulary ASR system. The AMIASR system [20] was used as the baseline system.

We began from the lattices for the whole AMI Meeting Corpus, generated by the AMIASR system using a trigram LM trained on a large set of data coming from Fisher, Hub4, Switchboard, webdata, and various meeting sources including AMI. We then generated 500-best lists from the lattices for each utterance. The reason why we used N-best rescoring instead of lattice rescoring is because the baseline lattices were generated using a trigram LM.

We adapted two LMs (Fisher, and AMI+Fisher+Hub4) trained in Section 4.1 according to the topic information extracted by HDP/rHDP models based on the previous ASR outputs, using a moving document window with a length of 10 seconds. The adapted LM was destroyed after it was used to rescore the current N-best lists. Two adapted LMs together with the baseline LM were then used to rescore the $\mathrm{N}$-best lists with a common language model weight of 14 (the same as for lattice generation) and no word insertion penalty.

Table 4.2 shows the WER results. LMs adapted by HDP/rHDP both yield an absolute reduction of about $0.7 \%$ in WER. This reduction is significant using a matched-pair significanc 4 test with $p<10^{-15}$. However, again there was no significant difference between the HDP and the rHDP.

To further investigate the power of HDP/rHDP-adapted LMs, we trained a standard unigram, AMI-1g, on the AMI training data, which is the same data

\footnotetext{
4 http://www.icsi.berkeley.edu/speech/faq/signiftest.html
} 
Table 2. The \%WER results of $\mathrm{HDP} / \mathrm{rHDP}$-adapted LMs

\begin{tabular}{|c||c|c|c||c|}
\hline LMs & SUB & DEL & INS & WER \\
\hline \hline Fisher & 22.7 & 11.4 & 5.8 & 39.9 \\
AMI-1g-adapted & 22.4 & 11.3 & 5.7 & 39.4 \\
HDP-adapted & 22.2 & 11.3 & 5.6 & 39.1 \\
rHDP-adapted & 22.3 & 11.3 & 5.6 & 39.2 \\
\hline \hline AMI+Fisher+Hub4 & 21.6 & 11.1 & 5.4 & 38.2 \\
AMI-1g-adapted & 21.3 & 11.0 & 5.4 & 37.8 \\
HDP-adapted & 21.2 & 11.1 & 5.3 & 37.6 \\
rHDP-adapted & 21.2 & 11.1 & 5.3 & 37.5 \\
\hline
\end{tabular}

used for $\mathrm{HDP} / \mathrm{rHDP}$ training. This unigram was trained using the same vocabulary of 7,910 words as that for $\mathrm{HDP} / \mathrm{rHDP}$ training. We then used this unigram as dynamic marginal to adapt the baseline LMs, also using the formula in Equation 9. The "AMI-1g-adapted" lines in Table 4.2 shows the WER results. We see, although AMI-1g-adapted LMs have lower WERs than that of the baseline LMs, HDP/rHDP-adapted LMs still have better WER performances (with $0.2-0.3 \%$ absolute reduction) than AMI-1g-adapted. Significant testing indicates that both improvements for the HDP/rHDP are significant, with $p<10^{-6}$.

\section{Discussion and Future Work}

In this paper, we successfully demonstrated the effectiveness of using the topic (and partly role) information to adapt LMs for ASR in meetings. The topics were automatically extracted using the nonparametric HDP model, which provides an efficient and flexible Bayesian framework for topic modeling. By defining the appropriate 'documents' for HDP models, we achieved a significant reduction in both perplexity and WER for a test set comprising about 11 hours of AMI meeting data.

To our understanding, the reasons for the significant improvements by adapted LMs based on the topic and role information via the HDP come from the following sources. First, the meeting corpus we worked on is a domain-specific corpus with limited vocabulary, especially for scenario meetings, with some words quite dominant during the meeting. So by roughly estimating the 'topic', and scaling those dominant words correctly, it is possible to improve LM accuracy. Second, HDP models can extract topics well, particularly on the domain-specific AMI Meeting Corpus. One interesting result we found is that different HDP/rHDP models, though trained using various different parameters, did not result in significant differences in either perplexity or WER. By closely looking at the resulting topics, we found that some topics have very high probability regardless of the different training parameters. One characteristic of those topics is that the top words normally have very high frequency. Third, the sentence-by-sentence style LM adaption provides further improvements, to those obtained using the AMI-1g-adapted LMs in Table 4.2. Language models are dynamically adapted 
according to the changes of topics detected based on the previous recognized results. This can be intuitively understood as a situation where there are $K$ unigram LMs, and we dynamically select one unigram to adapt the baseline LMs according to the context (topic). In this paper, however, both the number of unigram models $K$ and the unigram selected for a particular time are automatically determined by the HDP/rHDP. Although this is unsupervised adaptation, it performs better than LM adaptation using static LMs trained on reference data.

One the other hand, the rHDP had a similar accuracy to the HDP in terms of both perplexity and WER. Our interpretation for this is that we did not explicitly use the role information for adapting LMs, only using it as an additional DP level for sharing topics among different roles. As mentioned above, based on the AMI Meeting Corpus, which has a limited domain and consequently limited vocabulary words, this will not cause much difference in the resulting topics, no matter whether HDP or rHDP is used for topic modeling. Despite this, including the role information in the HDP framework can give us some additional information, such as the topics proportion specified to each role. This implies some scope to further incorporate role information into the hierarchical Bayesian framework for language modeling, for example by sampling the role randomly for each document, empirically analysing the differences between HDP and rHDP, and explicitly using the role for language modeling. Another possibility for further investigation is about the prior parameter for Dirichlet distribution: can prior knowledge from language be used to set this parameter? Finally, more ASR experiments to verify the consistence and significance of this framework on more meeting data, e.g., a 5-fold cross-validation on the AMI Meeting Corpus, would be informative.

\section{Acknowledgement}

We thank the AMI-ASR team for providing the baseline ASR system for experiments. This work is jointly supported by the Wolfson Microelectronics Scholarship and the European IST Programme Project FP6-033812 (AMIDA). This paper only reflects the authors' views and funding agencies are not liable for any use that may be made of the information contained herein.

\section{References}

1. Bengio, Y., Ducharme, R., Vincent, P., Jauvin, C.: A neural probabilistic language model. Journal of Machine Learning Research 3, 1137-1155 (2003)

2. Blitzer, J., Globerson, A., Pereira, F.: Distributed latent variable models of lexical co-occurrences. In: Proceedings of the 10th International Workshop on Artificial Intelligence and Statistics (2005)

3. Teh, Y.W.: A hierarchical Bayesian language model based on Pitman-Yor processes. In: Proc. of the Annual Meeting of the ACL, vol. 44 (2006)

4. Huang, S., Renals, S.: Hierarchical Pitman-Yor language models for ASR in meetings. In: Proc. IEEE Workshop on Automatic Speech Recognition and Understanding (ASRU 2007) (2007) 
5. Bilmes, J.A., Kirchhoff, K.: Factored language models and generalized parallel backoff. In: Proceedings of HLT/NACCL, pp. 4-6 (2003)

6. Xu, P., Emami, A., Jelinek, F.: Training connectionist models for the structured language model. In: Empirical Methods in Natural Language Processing, EMNLP 2003 (2003)

7. Wallach, H.M.: Topic modeling: Beyond bag-of-words. In: Proceedings of the 23rd International Conference on Machine Learning, Pittsburgh, PA (2006)

8. Carletta, J.: Unleashing the killer corpus: experiences in creating the multieverything AMI Meeting Corpus. Language Resources and Evaluation Journal 41(2), 181-190 (2007)

9. Teh, Y., Jordan, M., Beal, M., Blei, D.: Hierarchical Dirichlet processes. Journal of the American Statistical Association 101(476), 1566-1581 (2006)

10. Blei, D.M., Ng, A.Y., Jordan, M.I.: Latent Dirichlet allocation. Journal of Machine Learning Research 3 (2003)

11. Mrva, D., Woodland, P.C.: Unsupervised language model adaptation for Mandarin broadcast conversation transcription. In: Proc. of Interspeech (2006)

12. Tam, Y.C., Schultz, T.: Unsupervised LM adaptation using latent semantic marginals. In: Proc. of Interspeech (2006)

13. Hsu, B.J., Glass, J.: Style and topic language model adaptation using HMM-LDA. In: Proc. of EMNLP (2006)

14. Akita, Y., Nemoto, Y., Kawahara, T.: PLSA-based topic detection in meetings for adaptation of lexicon and language model. In: Proc. of Interspeech (2007)

15. Teh, Y.W.: Dirichlet processes. Encyclopedia of Machine Learning (Submitted, 2007)

16. Teh, Y.W., Kurihara, K., Welling, M.: Collapsed variational inference for HDP. Advances in Neural Information Processing Systems 20 (2008)

17. Ferguson, T.S.: A Bayesian analysis of some nonparametric problems. Annals of Statistics 1(2), 209-230 (1973)

18. Sethuraman, J.: A constructive definition of Dirichlet priors. Statistica Sinica 4, 639-650 (1994)

19. Kneser, R., Peters, J., Klakow, D.: Language model adaptation using dynamic marginals. In: Proc. of Eurospeech, Rhodes (1997)

20. Hain, T., et al.: The AMI system for the transcription of speech in meetings. In: Proc. of ICASSP 2007 (2007) 